\title{
Türkiye'nin Doğu Karadeniz Bölgesinde Yetişen Karayemiş Bitkisinin (Prunus laurocerasus L.) Meyve ve Toprak Örneklerinin Radyolojik Değerlendirilmesi
}

\author{
Radiological Evaluation of fruit and soil samples of Cherry Laurel Plant (Prunus \\ laurocerasus L.) Growing in the Eastern Black Sea Region of Turkey
}

\section{Erkan KIRIŞ*}

Recep Tayyip Erdoğan Üniversitesi, Fen Edebiyat Fakültesi, Fizik Bölümü, 53100, Rize

• Geliș tarihi / Received: 24.02.2018 • Düzeltilerek geliș tarihi / Received in revised form: 30.07.2018 • Kabul tarihi / Accepted: 10.08 .2018

$\ddot{O} \mathbf{z}$

$\mathrm{Bu}$ çalışma Doğu Karadeniz Bölgesinde on dört farklı istasyondan toplanan karayemiş (Prunus laurocerasus L.)'in meyve kısmında ve bu türün köklerindeki toprak örneklerinde ${ }^{226} \mathrm{Ra},{ }^{232} \mathrm{Th},{ }^{137} \mathrm{Cs}$ ve ${ }^{40} \mathrm{~K}$ radyonüklid konsantrasyon sonuçlarını göstermektedir. Karayemişin meyve kısmında ${ }^{226} \mathrm{Ra},{ }^{232} \mathrm{Th},{ }^{137} \mathrm{Cs}$ ve ${ }^{40} \mathrm{~K}$ 'ın ortalama aktivite konsantrasyonları sırasıyla 1.75, 1.03, 2.07 ve $215.38 \mathrm{~Bq} \mathrm{~kg}^{-1}$ (kuru ağırlık)'dır. Toprak örneklerinde ${ }^{226} \mathrm{Ra},{ }^{232} \mathrm{Th}$ ve ${ }^{40} \mathrm{~K}$ ’n ortalama aktivite konsantransyonları Dünya ortalaması değerlerinden daha düşük olarak belirlenmiştir. Karayemiş meyvesi ve toprak örneklerinde en yüksek ${ }^{137} \mathrm{Cs}$ radyonüklid konsantrasyonu Rize Merkez istasyonunda gözlemlenmiştir. Bunlara ek olarak, karayemişin tüketimine bağlı olarak ${ }^{226} \mathrm{Ra},{ }^{232} \mathrm{Th},{ }^{137} \mathrm{Cs}$ ve ${ }^{40} \mathrm{~K}$ 'ın günlük alımı, yıllık alınan etkin doz ve kanserojen risk değerleri hesaplanmış ve uluslararası değerlerle karşılaştırılmıştır. Ayrıca toprak örneklerinde radyum eşdeğer aktivitesi, soğurulmuş doz oranı, dış tehlike indeksi ve y1llık etkin doz eşdeğeri hesaplanmıştır. Bu hesaplamalara ilaveten topraktan karayemişin meyve kısmına geçen ${ }^{226} \mathrm{Ra},{ }^{232} \mathrm{Th}$, ${ }^{137} \mathrm{Cs}$ ve ${ }^{40} \mathrm{~K}$ radyonüklidleri için transfer faktörleri belirlenmiştir. Bu çalışmada elde edilen sonuçlar kanserojen risk ve radyolojik etki parametre değerlerinin herhangi bir risk taşımadığını göstermektedir.

Anahtar kelimeler: Günlük alım dozu, HPGe dedektör, Karayemiş, Radyoaktivite, Transfer faktörü

\begin{abstract}
This study presents the results of ${ }^{226} \mathrm{Ra},{ }^{232} \mathrm{Th},{ }^{137} \mathrm{Cs}$ and ${ }^{40} \mathrm{~K}$ radionuclide concentrations in the fruit part of cherry laurel (Prunus laurocerasus L.) and soil samples in root of this species were collected from fourteen different stations in the Eastern Black Sea Region. The mean activity concentrations of ${ }^{226} \mathrm{Ra},{ }^{232} \mathrm{Th},{ }^{137} \mathrm{Cs}$ and ${ }^{40} \mathrm{~K}$ in the fruit part of cherry laurel were 1.75, 1.03, 2.07 and $215.38 \mathrm{~Bq} \mathrm{~kg}^{-1}$, respectively. The mean activity concentrations of ${ }^{226} \mathrm{Ra},{ }^{232} \mathrm{Th}$ and ${ }^{40} \mathrm{~K}$ in soil samples were determined to be lower than the world average value. The highest ${ }^{137} \mathrm{Cs}$ radionuclide concentration in fruit of cherry laurel and soil samples was monitored in the Rize center location. In addition, radiological impact parameters such as daily intake of ${ }^{226} \mathrm{Ra},{ }^{232} \mathrm{Th},{ }^{137} \mathrm{Cs}$ and ${ }^{40} \mathrm{~K}$, annual committed effective dose and carcinogenic risk due to the consumption of cherry laurel were calculated and compared with the international data. The radium equivalent activity $\left(R a_{e q}\right)$, the absorbed dose rate $(D)$, the external hazard index $\left(H_{e x}\right)$ and the annual effective dose equivalent (AEDE) for soil samples were also evaluated. Moreover, transfer factors of ${ }^{226} \mathrm{Ra},{ }^{232} \mathrm{Th},{ }^{137} \mathrm{Cs}$ and ${ }^{40} \mathrm{~K}$ from soil to fruit part of cherry laurel were determined. The results indicate that the lifetime cancer risk and the radiological impact parameters values in the samples from the area studied in this present work is not significant.
\end{abstract}

Keywords: Daily intake dose, HPGe detector, Cherry laurel, Radioactivity, Transfer factor

*a Erkan KIRIŞ, erkan.kiris@erdogan.edu.tr, Tel: (0464) 2236126 (dâhili: 1867), orcid.org/0000-0003-2015-6005 


\section{Giriş}

Karayemiş (Prunus laurocerasus L. / Synonym: Laurocerasus officinalis Roem.) Rosaceae familyasına ait olup, Türkiye'de Karadeniz kıyılarında yayılış gösteren ve yerelde "Taflan" olarak adlandırılan beğenilerek tüketilen bir meyvedir (Alasalvar vd., 2005). Doğu Karadeniz Bölgesi'nde taze veya kurutulmuş şekilde yaygın olarak tüketilen karayemiş reçel, meyve suyu ve marmelat yapımında da kullanılmaktadır (Kolayli vd., 2003; Liyana-Pathirana vd., 2006).

Çevredeki radyoaktivite kaynakları doğal ve antropojenik kökenlere sahiptir (Badran vd., 2003). Doğal çevresel radyoaktivite ve öncelikle jeolojik ve coğrafi koşullara bağlı gama 1şınından dolayı maruz kalınan dış ışınlama dünyadaki her bölgenin topraklarında farklı seviyelerde görülmektedir (UNSCEAR, 2000). Doğal radyoaktivite temel olarak tüm yeryüzünün oluşumundan beri var olan ${ }^{40} \mathrm{~K},{ }^{238} \mathrm{U}$ ve ${ }^{232} \mathrm{Th}$ ve bunların bozunma ürünlerinden gelen radyonüklidlerden oluşmaktadır (Tzortzis vd., 2004). Yapay radyonüklidin büyük bir miktarı atmosferik nükleer silah testleri, Çernobil gibi nükleer kazalar sonucu serpinti olarak ve dünyay kirleten hava akımları ile birlikte çevreye salınmaktadır (Lu vd., 2006). ${ }^{137} \mathrm{Cs}, 30$ y1l yar1 ömrü olan antropojenik bir radyonükliddir. $\mathrm{Bu}$ yarı ömrün uzun olmas $1{ }^{137} \mathrm{Cs}$ radyonüklidini insan sağlığı için bir endişe kaynağı haline getirmektedir (Strode vd., 2012).

${ }^{238} \mathrm{U}$ ve ${ }^{232} \mathrm{Th}$ gibi doğal radyonüklidlerin radyoaktif bozunumu sonucu atmosferik hareketlerle çevreye yayılması ve çevrede her yerde bulunmaları ve kalıcı olmaları nedeniyle bu radyonüklidlerin aktivite konsantrasyonlarının belirlenmesi önemlidir (Pulhani vd., 2005). Toprakta doğal olarak oluşan uzun yarı ömürlü radyonüklidler, mineral alımı sırasında bitkiler üzerine transfer edilebilir ve bitkilerin çeşitli kısımlarında birikebilir. Böylece bu bitkilerin tüketimine bağlı olarak canlılar radyasyon dozuna maruz kalabilir (Shanthi vd., 2012).

Toprak-bitki sistemindeki kirleticilerin (radyonüklidler dahil) yer değiştirmesi ve birikimi karmaşıktır, ve değerlendirme modelleri yaygın olarak, besin zinciri boyunca radyonüklidlerin taşınmasını tahmin etmek için transfer faktörü (TF) olarak adlandırılan bir toprak-bitki konsantrasyon oranını kullanmaktadır. Bu oran topraktan bitkiye girmesi beklenen radyonüklid miktarını tanımlamaktadır (Chen vd., 2005). Belirli bir bitki türü ve radyonüklid için $\mathrm{TF}$, mevsim ve kirlilikten sonraki zamana bağlı olarak, bir alandan diğerine önemli ölçüde değişebilir. $\mathrm{Bu}$ değişiklikler toprağın fiziksel ve kimyasal özellikleri, çevresel koşullar ve topraktaki radyonüklidin kimyasal formu gibi çeşitli faktörlere bağlıdır (Persson ve Holm, 2011).

Radyoaktif kirlenme konusunda halk1 bilgilendirmek için doğal radyonüklid seviyelerini bilmenin yanı sıra, çevrede bulunan radyonüklidlerin yaydığ doz ve sağlık riskini değerlendirmek için doğal radyonüklidlerin dağılımını da ayrıca bilmek gereklidir (Lauria vd., 2009). Çevreye salınan radyoaktif kirliliğin izlenmesi çevreyi ve insan sağlığını korumak için çok önemlidir.

Bu çalışmada Doğu Karadeniz Bölgesi'nde yetişen karayemiş bitkilerinin meyvelerinde ve bu türün kökündeki toprak örneklerinde doğal ve yapay radyoaktivite seviyeleri belirlenmiştir. Ayrıca, karayemis meyvelerini tüketilmesine bağlı olarak ${ }^{226} \mathrm{Ra},{ }^{232} \mathrm{Th},{ }^{137} \mathrm{Cs}$ ve ${ }^{40} \mathrm{~K}$ ' $n$ radyolojik parametreleri hesaplanmış ve topraktan karayemişin meyve kısmına geçen ${ }^{226} \mathrm{Ra},{ }^{232} \mathrm{Th},{ }^{137} \mathrm{Cs}$ ve ${ }^{40} \mathrm{~K}$ radyonüklidleri için transfer faktörleri belirlenmiştir.

\section{Materyal ve Metot}

\section{1. Örnek Toplama}

Karayemiş örnekleri Temmuz 2013'de Doğu Karadeniz Bölgesi'ndeki on dört farkl1 istasyondan toplanmıştır. Karayemiş ağaçları çoğunlukla bahçelerin, evlerin ve yolların kenarlarında bulunmaktadır. Toprak örnekleri de aynı istasyonlardaki karayemiş ağaçlarının kökünden $0-10 \mathrm{~cm}$ derinlikten toplanmıştır. $\mathrm{Bu}$ çalışmadaki örnekleme alanları Türkiye'nin Karadeniz Kıyısının doğusunda yer alan Trabzon, Rize ve Artvin illeridir. Örnekleme istasyonlarının adları ve coğrafi koordinatları Tablo l'de verilmiştir.

\subsection{Radyoaktivite Ölçümleri}

Toplanan karayemiş örneklerinin meyve kısımları yapraklarından çıkartılmış ve sonra çekirdekleri meyve kısmından ayıklanmıștır. Meyve ve toprak örnekleri, nemi uzaklaştırmak için sicaklık kontrollü bir firında $85^{\circ} \mathrm{C}$ 'de 24 saat boyunca kurutulmuştur. Kuruyan örnekler ögütülmüş ve porselen havanda toz haline getirilmiştir. Her bir numune $63 \mu$ m'lik eleklerden geçirilerek homojen hale getirilmiş ve gama aktivite analizi için darası ve boş sayımları alınmış silindirik $100 \mathrm{~mL}$ 'lik plastik kaplara kapatılarak radyoaktif dengenin 
oluşması için 1 ay süreyle bekletilmiştir. Böylece örnekler gama spektrometresi cihazında sayıma hazır hale getirilmiştir.

Tablo 1. Örnekleme istasyonlarının isimleri ve coğrafi koordinatları

\begin{tabular}{lll}
\hline İstasyon & Enlem & Boylam \\
\hline Trabzon & & \\
Yomra & $40^{\circ} 57^{\prime} 14^{\prime \prime}$ & $39^{\circ} 52^{\prime} 00^{\prime \prime}$ \\
Araklı & $40^{\circ} 56^{\prime} 24^{\prime \prime}$ & $40^{\circ} 03^{\prime} 00^{\prime \prime}$ \\
Çamburnu & $40^{\circ} 54^{\prime} 42^{\prime \prime}$ & $40^{\circ} 12^{\prime} 10^{\prime \prime}$ \\
Sürmene & $40^{\circ} 54^{\prime} 40^{\prime \prime}$ & $40^{\circ} 07^{\prime} 08^{\prime \prime}$ \\
Of & $40^{\circ} 55^{\prime} 28^{\prime \prime}$ & $40^{\circ} 16^{\prime} 41^{\prime \prime}$ \\
Rize & & \\
İyidere & $41^{\circ} 00^{\prime} 41^{\prime \prime}$ & $40^{\circ} 21^{\prime} 37^{\prime \prime}$ \\
Derepazarı & $41^{\circ} 01^{\prime} 26^{\prime \prime}$ & $40^{\circ} 25^{\prime} 18^{\prime \prime}$ \\
Rize Merkez & $41^{\circ} 01^{\prime} 36^{\prime \prime}$ & $40^{\circ} 31^{\prime} 02^{\prime \prime}$ \\
Çayeli & $41^{\circ} 04^{\prime} 58^{\prime \prime}$ & $40^{\circ} 42^{\prime} 58^{\prime \prime}$ \\
Pazar & $41^{\circ} 10^{\prime} 49^{\prime \prime}$ & $40^{\circ} 53^{\prime} 11^{\prime \prime}$ \\
Ardeşen & $41^{\circ} 11^{\prime} 30^{\prime \prime}$ & $40^{\circ} 59^{\prime} 18^{\prime \prime}$ \\
Fındıkl & $41^{\circ} 16^{\prime} 14^{\prime \prime}$ & $41^{\circ} 08^{\prime} 23^{\prime \prime}$ \\
Artvin & & \\
Arhavi & $41^{\circ} 21^{\prime} 10^{\prime \prime}$ & $41^{\circ} 18^{\prime} 31^{\prime \prime}$ \\
Hopa & $41^{\circ} 23^{\prime} 40^{\prime \prime}$ & $41^{\circ} 25^{\prime} 11^{\prime \prime}$ \\
\hline
\end{tabular}

Örneklerin radyoaktiflik ölçümleri için 1332.5 keV'de $1.9 \mathrm{keV}$ ayırma gücüne ve $\% 55^{\prime}$ 'lik relatif verime sahip ORTEC marka GEM55P4-95 model HPGe detektörü kullanılmıștır. HPGe detektörün enerji ve verim kalibrasyonu yapıldıktan sonra, temin ettiğimiz yosun-toprak (IAEA-447) radyoaktif referans malzemesi sistemde 3 tekrarlı olarak okutuldu ve ortalaması alınarak cihazın doğruluğu tespit edildi. Radyoaktif dengenin sağlanmas1 için bir aylık bekleme süresinden sonra ölçümlere geçildi. Sayımlarda kullanılan HPGe dedektörünün besleme voltajı yaklaşık 5000 V'tur. Sayım programı olarak Genie-2000 kullanıldı. Örnekler, dedektörün hemen önündeki kurşunla kaplı numune odasına konuldu. Numune sayım süresi karayemiş örnekleri için $100000 \mathrm{~s}$ ve toprak örnekleri için $50000 \mathrm{~s}$ olarak seçildi. Bu süre sonunda numunelerden yayınlanan radyoaktif izotoplara ait spektrumlar elde edildi. Dedektör çevresindeki ortamdaki arka plan dağılımını belirlemek için boş bir kap, örneklerle aynı şekilde ve geometride sayıldı. Arka plan spektrumu, ölçülen izotopların gama 1şınlarının net pik alanını düzeltmek için kullanıldı (Baltas vd., 2014).

Örneklerin spesifik aktiviteleri \%3-7 düzeyindeki hatalar içinde sertifikalı değerlerine uygun olarak elde edilmiştir. ${ }^{226} \mathrm{Ra}$ serisinin aktivite konsantrasyonunu belirlemek için $351.9 \mathrm{keV}$ $\left({ }^{214} \mathrm{~Pb}\right)$ ve $609.3 \mathrm{keV}\left({ }^{214} \mathrm{Bi}\right)$ enerjilerin gama-1şını geçişleri kullanıldı. ${ }^{232} \mathrm{Th}$ serisinin aktivite konsantrasyonunu belirlemek için $911.1 \mathrm{keV}$ $\left({ }^{228} \mathrm{Ac}\right)$ ve $583.1 \mathrm{keV}\left({ }^{208} \mathrm{Tl}\right)$ gama 1 şını çizgileri kullanıldı. ${ }^{40} \mathrm{~K}$ ve ${ }^{137} \mathrm{Cs}$ aktivite konsantrasyonları belirlemek için sırasıyla 1460.8 ve $661.6 \mathrm{keV}$ gama 1şını geçişleri kullanılmıştır.

Ölçülen örneklerdeki radyonüklidlerin aktivite konsantrasyonları aşağıdaki denklem kullanılarak hesaplanmıştır:

$\mathrm{C}=\frac{\mathrm{N}}{\varepsilon \cdot \mathrm{P}_{\gamma} \cdot \mathrm{T} \cdot \mathrm{m}}\left(\mathrm{Bq} \mathrm{kg}^{-1}\right)$

Burada $\mathrm{N}$ gama 1şınının sayım sayısı, $\varepsilon$ ilgili gama enerjisindeki verim, $P_{\gamma}$ gama ışınının salınım hızı, $\mathrm{T}$ sayma süresi ve $\mathrm{m}$ numune miktarıdır.

Minimum dedekte edilebilir aktivite, belli koşullar altında ölçüm sisteminin dedeksiyon kapasitesini ifade etmek için kullanılan bir terimdir. Minimum dedekte edilebilir aktivite (MDA) aşağıdaki formül kullanılarak hesaplanmıştır (Currie, 1968):

$\mathrm{MDA}=\frac{\sigma \sqrt{\mathrm{B}}}{\varepsilon \cdot \mathrm{P} \gamma \cdot \mathrm{T} \cdot \mathrm{m}}$

Burada MDA $\mathrm{Bq} \mathrm{kg}^{-1}$ biriminde olup, $\sigma$ istatistiksel kapsama faktörüdür ve 1.645 değerine eşittir, $\mathrm{B}$ ise artalan radyasyon değeridir. MDA değerleri ${ }^{226} \mathrm{Ra}$ için $0.16 \mathrm{~Bq} \mathrm{~kg}^{-1},{ }^{232} \mathrm{Th}$ için 0.24 $\mathrm{Bq} \mathrm{kg}{ }^{-1},{ }^{137} \mathrm{Cs}$ için $0.02 \mathrm{~Bq} \mathrm{~kg}^{-1}$ ve ${ }^{40} \mathrm{~K}$ için 1.69 $\mathrm{Bq} \mathrm{kg}{ }^{-1}$ olarak hesaplanmıştır. Yosun-toprak (IAEA-447) radyoaktif referans malzemesi için aktivite konsantrasyonlarının sertifa değerleri sırasıyla ${ }^{226} \mathrm{Ra}$ için $25.04 \mathrm{~Bq} \mathrm{~kg}^{-1}$, ${ }^{232} \mathrm{Th}$ için 37.3 $\mathrm{Bq} \mathrm{kg}{ }^{-1},{ }^{137} \mathrm{Cs}$ için $371.11 \mathrm{~Bq} \mathrm{~kg}{ }^{-1}$ ve ${ }^{40} \mathrm{~K}$ için 550 $\mathrm{Bq} \mathrm{kg}{ }^{-1}$ değerlerine eşittir. Kullanılan dedektör sisteminde bu değerler ${ }^{226} \mathrm{Ra}$ için $23.96 \mathrm{~Bq} \mathrm{~kg}^{-1}$, ${ }^{232} \mathrm{Th}$ için $35.7 \mathrm{~Bq} \mathrm{~kg}^{-1},{ }^{137} \mathrm{Cs}$ için $362.55 \mathrm{~Bq} \mathrm{~kg}{ }^{-1}$ ve ${ }^{40} \mathrm{~K}$ için $521 \mathrm{~Bq} \mathrm{~kg}^{-1}$ olarak belirlenmiştir.

\subsection{Radyonüklidlerin Günlük Alımı}

Radyoaktivitenin günlük alımı karayemişin tüketilmesiyle insan vücuduna alınan ${ }^{226} \mathrm{Ra},{ }^{232} \mathrm{Th}$, ${ }^{137} \mathrm{Cs}$ ve ${ }^{40} \mathrm{~K}$ radyonüklidlerin birikmesi olarak düşünülmektedir. Rize'de günde kişi başına 
karayemiş tüketimi $2.74 \mathrm{~g}$ olarak verilmiştir (Korkmaz Görür vd., 2012). Karayemişin tüketilmesinden dolayı insanların vücutlarına biriktirdiği radyoaktivitenin günlük alımı aşağıdaki formülle hesaplanmıştır (Khandaker vd., 2013):

$\mathrm{GA}=\frac{\mathrm{C} \times \mathrm{A}_{\mathrm{ig}} \times \mathrm{F}_{\mathrm{c}}}{\mathrm{Y}_{\mathrm{d}}}$

Burada, GA insanlar tarafından radyonüklilerin günlük alımı $\left(\mathrm{Bq}\right.$ gün $\left.{ }^{-1}\right), \mathrm{C}$ radyonüklidlerin aktivite konsantrasyonu $\left(\mathrm{Bq} \mathrm{kg}{ }^{-1}\right), \mathrm{A}_{\mathrm{ig}}$ yılda kişi başına karayemiş tüketimi, $F_{c}$ tüketim kesri ve $Y_{d}$ bir yıldaki gün sayısıdır.

\subsection{Yillık Alınan Etkin Doz}

Karayemişin tüketilmesiyle ${ }^{226} \mathrm{Ra},{ }^{232} \mathrm{Th},{ }^{137} \mathrm{Cs}$ ve ${ }^{40} \mathrm{~K}$ radyonüklidlerin vücuda alınmasından dolayı insanlara geçen yıllık etkin doz aşağıdaki denklemle hesaplanmıştır (Khandaker vd., 2013):

$\operatorname{YAED}\left(\operatorname{Svyl}{ }^{-1}\right)=\mathrm{C} \times \mathrm{A}_{\mathrm{ig}} \times \mathrm{D}_{\mathrm{cf}} \times \mathrm{F}_{\mathrm{c}}$

Burada, YAED radyonüklidlerin vücuda alınmasıyla insanlara geçen yıllık etkin doz, C radyonüklidlerin ortalama aktivite konsantrasyonu $\left(\mathrm{Bq} \mathrm{kg}^{-1}\right), \mathrm{A}_{\mathrm{ig}}$ karayemişin yıllık tüketimi $\left(\mathrm{kg} \mathrm{yil}^{-1}\right)$ ve $D_{c f}$ ilgili radyonüklidin doz alım dönüşüm faktörü' dür $\left({ }^{226} \mathrm{Ra}\right.$ için $2.8 \times 10^{-7} \mathrm{~Sv} \mathrm{~Bq}^{-1},{ }^{232} \mathrm{Th}$ için $2.3 \times 10^{-7} \mathrm{~Sv} \mathrm{~Bq}^{-1},{ }^{137} \mathrm{Cs}$ için $1.3 \times 10^{-8} \mathrm{~Sv} \mathrm{~Bq}^{-1}$ ve $^{40} \mathrm{~K}$ için $6.2 \times 10^{-9} \mathrm{~Sv} \mathrm{~Bq}^{-1}$ ) (IAEA, 2011). ${ }^{226} \mathrm{Ra},{ }^{232} \mathrm{Th}$ ve ${ }^{40} \mathrm{~K}$ radyonüklidleri için Dünya ortalamas1 değerleri sirasiyla 120,120 ve $170 \mu \mathrm{Sv} \mathrm{y}^{-1}$ olarak verilmiştir (UNSCEAR, 2000). Toplam yıllık alınan etkin doz ise aşağıdaki formül ile hesaplanmıştır (Khandaker vd., 2013).

$\mathrm{YAED}_{\mathrm{T}}=\left(\mathrm{C}_{\mathrm{Ra}}+\mathrm{C}_{\mathrm{Th}}+\mathrm{C}_{\mathrm{Cs}}+\mathrm{C}_{\mathrm{K}}\right) \times \mathrm{A}_{\mathrm{ig}} \times \mathrm{D}_{\mathrm{cf}}$

Burada $\mathrm{C}_{\mathrm{Ra}}, \mathrm{C}_{\mathrm{Th}}, \mathrm{C}_{\mathrm{Cs}}$ ve $\mathrm{C}_{\mathrm{K}}$ sirasiyla ${ }^{226} \mathrm{Ra},{ }^{232} \mathrm{Th}$, ${ }^{137} \mathrm{Cs}$ ve ${ }^{40} \mathrm{~K}$ radyonüklidlerinin aktivite konsantrasyonlarıdır.

\subsection{Kanserojen Risk}

Daha fazla radyasyona maruz kalma durumu kanser sıklığının artması ile ilişkilendirilerek, karayemiş gibi gıda örneklerinin yenmesi durumunda kanser riski belirlenebilir. Kanser riskini hesaplamak için aşağıdaki denklem (Khandaker vd., 2015) kullanılmıştır.

$\mathrm{KR}=\mathrm{A}_{\mathrm{ir}} \times \mathrm{A}_{\mathrm{ls}} \times \mathrm{R}_{\mathrm{c}}$
Burada $\mathrm{KR}, \mathrm{A}_{\mathrm{ir}}, \mathrm{A}_{\mathrm{ls}}$ ve $\mathrm{R}_{\mathrm{c}}$ sirasiyla kanser riski, radyonüklidin y1llık alımı $(\mathrm{Bq})$, ortalama yaşam süresi $(78 \mathrm{y})$ ve ölüm riski katsayısıdır $\left(\mathrm{Bq}^{-1}\right)$. USEPA'dan alınan ölüm riski katsayısı değerleri ${ }^{226} \mathrm{Ra}$ için $9.56 \times 10^{-9} \mathrm{~Bq}^{-1},{ }^{232} \mathrm{Th}$ için $2.45 \times 10^{-9} \mathrm{~Bq}^{-1}$, ${ }^{137} \mathrm{Cs}$ için $9.25 \times 10^{-13} \mathrm{~Bq}^{-1}$ ve ${ }^{40} \mathrm{~K}$ için $5.89 \times 10^{-10}$ $\mathrm{Bq}^{-1}$ dir (USEPA, 1999). Kanserojen risk için kabul edilen limit değerinin $10^{-3}$ olduğu belirtilmiştir (Khandaker vd., 2015).

\subsection{Radyum Eşdeğer Aktifliği}

${ }^{226} \mathrm{Ra},{ }^{232} \mathrm{Th}$ ve ${ }^{40} \mathrm{~K}$ 'ın numunelerdeki dağ 1 lımı birbirlerinden farklıdır. Farklı miktarlardaki bu spesifik aktifliklerinden radyasyona maruz kalmayı standartlaştırmak için radyum eşdeğer aktifliği $\left(\mathrm{Ra}_{\mathrm{eq}}\right)$ aşağıdaki ifade ile tanımlanmıştır (Beretka ve Mathew, 1985; UNSCEAR, 2000; Amrani ve Tahtat, 2001):

$\mathrm{Ra}_{\mathrm{eq}}=\mathrm{C}_{\mathrm{Ra}}+1.43 \mathrm{C}_{\mathrm{Th}}+0.077 \mathrm{C}_{\mathrm{K}}$

Burada $C_{R a}, C_{T h}$ ve $C_{K}$ sirasiyla ${ }^{226} R a,{ }^{232} T h$ ve ${ }^{40} \mathrm{~K}^{\prime} \mathrm{n} \quad \mathrm{Bq} \quad \mathrm{kg}^{-1}$ biriminde spesifik aktivite değerleridir.

\subsection{Soğurulan Gama Doz Huzı}

Yeryüzünün $1 \mathrm{~m}$ üzerinde havada soğrulmuş doz oranı aşağıdaki formülle belirlenir (UNSCEAR, 2000).

$\mathrm{D}\left(\right.$ n Gy saat $\left.^{-1}\right)=0,462 \mathrm{C}_{\mathrm{Ra}}+0,604 \mathrm{C}_{\mathrm{Th}}+$ $0,0417 \mathrm{C}_{\mathrm{K}}$

Burada $C_{R a}, C_{T h}$ ve $C_{K}$ sirasiyla ${ }^{226} R a,{ }^{232} T h$ ve ${ }^{40} \mathrm{~K}^{\prime} \mathrm{in} \mathrm{Bq} \mathrm{kg}{ }^{-1}$ biriminde spesifik aktiviteleridir. ${ }^{137} \mathrm{Cs}$ çevreyle ilgili arka plandan toplam doza dahil edilememesi nedeniyle burada göz ardı edilmiştir (Amanjeet vd., 2017).

\subsection{Dış Tehlike İndeksi}

Dış tehlike indeksi $\mathrm{H}_{\mathrm{ex}}$, Krieger (Krieger, 1981) tarafindan önerilen model kullanılarak, incelenen örnekler için hesaplanmıştır; pencere ve kapılar olmadan kalın duvarlar varsayımıyla, dış tehlike indeksi aşağıdaki formülle belirlenmiştir.

$\mathrm{H}_{\mathrm{ex}}=\frac{\mathrm{C}_{\mathrm{Ra}}}{370}+\frac{\mathrm{C}_{\mathrm{Th}}}{259}+\frac{\mathrm{C}_{\mathrm{K}}}{4810} \leq 1$

Burada $\mathrm{C}_{\mathrm{Ra}}, \mathrm{C}_{\mathrm{Th}}$ ve $\mathrm{C}_{\mathrm{K}}$ sirasiyla ${ }^{226} \mathrm{Ra},{ }^{232} \mathrm{Th}$ ve ${ }^{40} \mathrm{~K}^{\prime}$ in Bq kg ${ }^{-1}$ biriminde spesifik aktiviteleridir. 


\subsection{Yillk Etkin Doz}

Soğrulan doz için dönüşüm faktörü $\left(0.7 \mathrm{~Sv} \mathrm{~Gy}{ }^{-1}\right)$ ve dış ortamlarda maruz kalma faktörü $(0.2)$ (UNSCEAR, 2000) dikkate alınarak etkin doz aşağıdaki formülle hesaplanmıştır.

YED $\left(\mu \mathrm{Sv} \mathrm{yll}^{-1}\right)$

$=\mathrm{D}\left(\right.$ nGy saat $\left.^{-1}\right) \times 8760\left(\right.$ saat yll $\left.^{-1}\right) \times 0.2 \times 0.7 \mathrm{~Sv} \mathrm{~Gy}^{-1} \times 10^{-3}$

\subsection{Transfer Faktörï}

Transfer faktörü (TF) kök sistemi yoluyla radyonüklidlerin $\left({ }^{226} \mathrm{Ra},{ }^{232} \mathrm{Th},{ }^{137} \mathrm{Cs}\right.$ ve $\left.{ }^{40} \mathrm{~K}\right)$ topraktan bitkiye geçişini göstermek için kullanılır. TF değerleri bitki ve bitki kökündeki toprak örnekleri için elde edilen radyonüklidlerin aktivite konsantrasyonları kullanılarak aşağıdaki formülle hesaplanmıştır (IUR, 1994).

$\mathrm{TF}=\frac{\mathrm{C}_{\mathrm{p}}}{\mathrm{C}_{\mathrm{s}}}$
Burada $\mathrm{C}_{\mathrm{p}}$ bitki kısmındaki radyonüklid aktivitesi $\left(\mathrm{Bq} \mathrm{kg}^{-1}\right.$ ) ve $\mathrm{C}_{\mathrm{s}}$ ise topraktaki radyonüklid aktivitesidir $\left(\mathrm{Bq} \mathrm{kg}^{-1}\right)$.

\section{Bulgular ve Tartışma}

Çalışma alanı olarak on dört farklı istasyondan toplanan karayemiş örneklerinin meyve kısımlarında ve bu türün köklerindeki toprak örneklerinde doğal ve yapay radyonüklidlerinin aktivite konsantrasyonları belirlenmiştir. Karayemiş için bu konsantrasyon değerleri Tablo 2 'de verilmiştir. Karayemişin meyve kısmında aktivite konsantrasyon aralıkları ${ }^{226} \mathrm{Ra}$ için $0.22 \pm 0.01-5.17 \pm 0.28 \mathrm{~Bq} \mathrm{~kg}{ }^{-1},{ }^{232} \mathrm{Th}$ için $0.43 \pm 0.02-2.18 \pm 0.12 \mathrm{~Bq} \mathrm{~kg}{ }^{-1},{ }^{137} \mathrm{Cs}$ için $0.27 \pm 0.01-10.28 \pm 0.42 \mathrm{~Bq} \mathrm{~kg}{ }^{-1}$ ve ${ }^{40} \mathrm{~K}$ için $170.98 \pm 6.15$ - 270.09 $\pm 11.07 \mathrm{~Bq} \mathrm{~kg}{ }^{-1}$ olarak belirlenmiştir. Bu sonuçlara göre en yüksek ${ }^{226} \mathrm{Ra}$, ${ }^{232} \mathrm{Th},{ }^{137} \mathrm{Cs}$ ve ${ }^{40} \mathrm{~K}$ konsantrasyon değerleri sirasiyla Pazar, Hopa, Rize merkez ve Sürmene istasyonlarında gözlemlenmiştir.

Tablo 2. Karayemişin ${ }^{226} \mathrm{Ra},{ }^{232} \mathrm{Th},{ }^{137} \mathrm{Cs}$ ve ${ }^{40} \mathrm{~K}$ aktivite konsantrasyonları.

\begin{tabular}{|c|c|c|c|c|}
\hline \multirow{2}{*}{ İstasyon } & \multicolumn{4}{|c|}{ Aktivite Konsantrasyonları (Bq kg $\left.{ }^{-1}\right)$} \\
\hline & ${ }^{226} \mathbf{R a}$ & ${ }^{232} \mathrm{Th}$ & ${ }^{137} \mathrm{Cs}$ & ${ }^{40} K$ \\
\hline \multicolumn{5}{|l|}{ Trabzon } \\
\hline Yomra & $<\mathrm{DL}$ & $0.86 \pm 0.04$ & $0.76 \pm 0.04$ & $226.12 \pm 10.87$ \\
\hline Araklı & $2.67 \pm 0.11$ & $0.88 \pm 0.05$ & $<\mathrm{DL}$ & $215.24 \pm 10.44$ \\
\hline Çamburnu & $0.22 \pm 0.01$ & $0.77 \pm 0.04$ & $<\mathrm{DL}$ & $176.88 \pm 7.51$ \\
\hline Sürmene & $0.39 \pm 0.02$ & $1.44 \pm 0.07$ & $<\mathrm{DL}$ & $270.09 \pm 11.07$ \\
\hline Of & $<\mathrm{DL}$ & $<\mathrm{DL}$ & $<\mathrm{DL}$ & $177.22 \pm 8.15$ \\
\hline \multicolumn{5}{|l|}{ Rize } \\
\hline İyidere & $0.43 \pm 0.02$ & $<\mathrm{DL}$ & $1.24 \pm 0.06$ & $262.64 \pm 10.75$ \\
\hline Derepazarı & $0.43 \pm 0.02$ & $0.95 \pm 0.05$ & $0.37 \pm 0.02$ & $182.32 \pm 7.02$ \\
\hline Rize Merkez & $<\mathrm{DL}$ & $<\mathrm{DL}$ & $10.28 \pm 0.42$ & $179.06 \pm 9.08$ \\
\hline Çayeli & $<\mathrm{DL}$ & $0.94 \pm 0.05$ & $3.47 \pm 0.18$ & $260.39 \pm 13.21$ \\
\hline Pazar & $5.17 \pm 0.28$ & $0.87 \pm 0.21$ & $0.27 \pm 0.01$ & $234.81 \pm 10.95$ \\
\hline Ardeşen & $1.98 \pm 0.09$ & $<\mathrm{DL}$ & $0.68 \pm 0.03$ & $243.64 \pm 10.59$ \\
\hline Findıklı & $0.90 \pm 0.05$ & $0.43 \pm 0.02$ & $1.83 \pm 0.09$ & $170.98 \pm 6.15$ \\
\hline \multicolumn{5}{|l|}{ Artvin } \\
\hline Arhavi & $0.88 \pm 0.04$ & $<\mathrm{DL}$ & $0.51 \pm 0.03$ & $222.30 \pm 9.38$ \\
\hline Hopa & $4.45 \pm 0.17$ & $2.18 \pm 0.12$ & $1.25 \pm 0.06$ & $193.61 \pm 7.68$ \\
\hline Ortalama & 1.75 & 1.03 & 2.07 & 215.38 \\
\hline Min.-Mak. & $0.22-5.17$ & $0.43-2.18$ & $0.27-10.28$ & $170.98-270.09$ \\
\hline
\end{tabular}

$<$ DL: Dedeksiyon limitinin altında 
Karayemiş örnekleri için belirlenen ortalama ${ }^{226} \mathrm{Ra},{ }^{232} \mathrm{Th},{ }^{137} \mathrm{Cs}$ ve ${ }^{40} \mathrm{~K}$ aktivite değerleri daha önce yapılan çalışmayla karşılaştırıldığı zaman Rize'de (Korkmaz Görür vd., 2012) karayemiş için elde edilen ortalama verilerden $\left({ }^{238} \mathrm{U}\left({ }^{226} \mathrm{Ra}\right)\right.$, ${ }^{232} \mathrm{Th},{ }^{137} \mathrm{Cs}$ ve ${ }^{40} \mathrm{~K}$ için sirasıyla $0.72,1.64,2.46$ ve $222.24 \mathrm{~Bq} \mathrm{~kg}^{-1}$ ) ${ }^{226} \mathrm{Ra}$ hariç ${ }^{232} \mathrm{Th},{ }^{137} \mathrm{Cs}$ ve ${ }^{40} \mathrm{~K}$ 'ın ortalama değerlerinin daha düşük olduğu ve üzüm için elde edilen ortalama verilerden $\left({ }^{238} \mathrm{U}\right.$ $\left({ }^{226} \mathrm{Ra}\right),{ }^{232} \mathrm{Th},{ }^{137} \mathrm{Cs}$ ve ${ }^{40} \mathrm{~K}$ için sirasıyla 8.04 , $3.22,1.78$ ve $\left.369.71 \mathrm{~Bq} \mathrm{~kg}^{-1}\right){ }^{137} \mathrm{Cs}$ hariç ${ }^{226} \mathrm{Ra}$, ${ }^{232} \mathrm{Th}$ ve ${ }^{40} \mathrm{~K}$ 'ın ortalama değerlerinin daha düşük olduğu tespit edilmiştir. Karayemişin tüketilmesiyle radyonüklidlerin vücuda alınmasından dolayı insana geçen ${ }^{226} \mathrm{Ra},{ }^{232} \mathrm{Th}$, ${ }^{137} \mathrm{Cs}$ ve ${ }^{40} \mathrm{~K}$ 'ın günlük alımı, yıllık alınan etkin doz, toplam ylllık alınan etkin doz ve kanserojen risk değerleri Tablo 3'de verilmiştir. Tahmin edilen y1llık etkin doz değerlerinin Dünya ortalaması değerlerinden daha düşük olduğu tespit edilmiştir. ${ }^{226} \mathrm{Ra},{ }^{232} \mathrm{Th}$ ve ${ }^{40} \mathrm{~K}$ radyonüklidlerinin alımı sonucu yıllık etkin doz değerlerinin Dünya ortalamaları sirasiyla 120,120 ve $170 \mu \mathrm{Sv} \mathrm{y}_{1} 1^{-1}$ dir (UNSCEAR, 2000). Kanserojen risk değerlerinin de kabul edilen kanser riski değerinden $\left(10^{-3}\right)$ (Khandaker vd., 2015) daha düşük olduğu belirlenmiştir.

Karayemiş türlerinin köklerinden toplanan toprak örnekleri için belirlenen ${ }^{226} \mathrm{Ra},{ }^{232} \mathrm{Th},{ }^{137} \mathrm{Cs}$ ve ${ }^{40} \mathrm{~K}$ 'ın aktivite konsantrasyonları, radyum eşdeğer aktiviteleri, soğurulan doz oranları, dış tehlike indeksleri ve y1llık etkin doz oranlar1 Tablo 4 'te verilmiştir. Toprak örneklerinde aktivite konsantrasyon aralıkları ${ }^{226} \mathrm{Ra}$ için $5.21 \pm 0.25$ $32.93 \pm 1.28 \mathrm{~Bq} \mathrm{~kg}^{-1},{ }^{232} \mathrm{Th}$ için $6.18 \pm 0.32 \quad-$ $42.62 \pm 1.84 \mathrm{~Bq} \mathrm{~kg}{ }^{-1},{ }^{137} \mathrm{Cs}$ için $2.80 \pm 0.15-$ $368.11 \pm 20.24 \mathrm{~Bq} \mathrm{~kg}^{-1}$ ve ${ }^{40} \mathrm{~K}$ için $146.66 \pm 5.82-$ $650.94 \pm 38.52 \mathrm{~Bq} \mathrm{~kg}$ olarak belirlenmiştir. Toprak örneklerinde en yüksek ${ }^{226} \mathrm{Ra}$ ve ${ }^{232} \mathrm{Th}$ konsantrasyonu Hopa istasyonunda izlenirken, ${ }^{137} \mathrm{Cs}$ ve ${ }^{40} \mathrm{~K}$ 'nın en yüksek konsantrasyonu sırasıyla Sürmene ve Pazar istasyonlarında izlenmiştir. Toprak örnekleri için elde edilen ${ }^{226} \mathrm{Ra},{ }^{232} \mathrm{Th}$ ve ${ }^{40} \mathrm{~K}$ 'n ortalama aktivite konsantrasyonlarının Dünya ortalamas1 değerlerinden daha düşük olduğu tespit edilmiştir. Toprak için ${ }^{226} \mathrm{Ra},{ }^{232} \mathrm{Th}$ ve ${ }^{40} \mathrm{~K}$ 'n Dünya ortalaması değerleri sirasıyla 35,30 ve $400 \mathrm{~Bq} \mathrm{~kg}^{-}$ ${ }^{1}$, dir. (UNSCEAR, 2000).

Toprak örnekleri için elde edilen en yüksek ${ }^{226} \mathrm{Ra}$, ${ }^{232} \mathrm{Th}$ ve ${ }^{40} \mathrm{~K}$ aktivite değerleri daha önce yapılan çalışmalarla karşılaştırıldığı zaman Rize (Fırtına Vadisi) (Kurnaz vd., 2007), Trabzon (Kurnaz vd., 2011), Giresun (Celik vd., 2008) ve Doğu Karadeniz Bölgesi (Celik vd., 2009) için elde edilen en yüksek verilerden (sırasıyla ${ }^{226} \mathrm{Ra}$ için 188.26, $140\left({ }^{238} \mathrm{U}\right), 85$ ve $120.3 \mathrm{~Bq} \mathrm{~kg}^{-1},{ }^{232} \mathrm{Th}$ için 105.23, 116,82 ve $121.4 \mathrm{~Bq} \mathrm{~kg}^{-1}$ ve ${ }^{40} \mathrm{~K}$ için 1234.65, 1697, 1301 ve $1294.5 \mathrm{~Bq} \mathrm{~kg}^{-1}$ ) daha düşük olduğu gözlemlenmiştir. Araklı'da ölçülen en yüksek ${ }^{40} \mathrm{~K}$ aktivite değeri Araklı'daki toprak örnekleri için yapılan çalışmalarla (Celik vd., 2009; Kurnaz vd., 2011) karşılaştırıldı̆̆1 zaman elde edilen aktivite değerlerinden (sirasıyla 348.9 - $547.8 \mathrm{~Bq} \mathrm{~kg}^{-1}$ ve $389 \mathrm{~Bq} \mathrm{~kg}^{-1}$ ) daha yüksek olduğu tespit edilmiştir. ${ }^{40} \mathrm{~K}$ aktivite değerindeki bu artış bu alanlarda uzun vadede yapılan mineral gübreleme uygulamasıyla bağlantılı olabilir (Yazici vd., 2008). Elde edilen en yüksek ${ }^{137} \mathrm{Cs}$ aktivite değeri ise Giresun (Celik vd., 2008) ve Doğu Karadeniz Bölgesi (Celik vd., 2009) için elde edilen en yüksek değerlerden (sırasıyla 1304 ve $774.7 \mathrm{~Bq} \mathrm{~kg}{ }^{-1}$ ) daha düşük olduğu, Rize (Frrtına Vadisi) (Kurnaz vd., 2007) ve Trabzon (Kurnaz vd., 2011) için elde edilen en yüksek değerlerden (surasıyla 232.44 ve $199 \mathrm{~Bq} \mathrm{~kg}^{-1}$ ) daha yüksek olduğu tespit edilmiştir. Rize Merkez istasyonunun (Fener Mahallesi) toprak örneğinde ölçülen en yüksek ${ }^{137} \mathrm{Cs}$ aktivite değerinin Rize'de yapılan bir çalışmayla (Keser vd., 2011) karşılaştırıldığı zaman bitki köklerindeki toprak örnekleri için elde edilen en yüksek veriden (578.6 $\mathrm{Bq} \mathrm{kg} \mathrm{kg}^{-1}$ ) daha düşük olduğu tespit edilmiştir. Ölçülen ${ }^{137} \mathrm{Cs}$ radyonüklidinin, birkaç ülke tarafindan gerçekleştirilen atmosferik nükleer silah testlerinden kaynaklandığ 1 düşünülmektedir; Bununla birlikte, küçük bir miktarının da 1986'da meydana gelen Çernobil kazasından kaynaklı olabileceği düşünülmektedir (Baltas vd., 2017) ve serpinti şeklinde yayılan radyasyonun çoğu toprakta birikebileceği de ifade edilmektedir (UNSCEAR, 1982). Ayrica, Rize Merkez istasyonunun karayemiş örneğinde ${ }^{137} \mathrm{Cs}$ aktivite değerinin yüksek olması, bu bitki kökündeki toprak örneğinde ${ }^{137} \mathrm{Cs}$ aktivite değerinin yüksek olmasına dayandırılabilir.

Toprak örnekleri için hesaplanan $\mathrm{Ra}_{\mathrm{eq}}$ değerlerinin $33.8-126.60 \mathrm{~Bq} \mathrm{~kg}{ }^{-1}$ aralığında değiştiği ve ortalama $\mathrm{Ra}_{\text {eq }}$ değerinin $72.67 \mathrm{~Bq} \mathrm{~kg}{ }^{-1}$ olduğu belirlenmiştir. Hesaplanan bu ortalama değerin tavsiye edilen maksimum $\mathrm{Ra}_{\text {eq }}$ değerinden (370 $\mathrm{Bq} \mathrm{kg}^{-1}$ ) (UNSCEAR, 2000) daha düşük olduğu tespit edilmiştir. Soğurulan doz oranları değerlerinin 16.24-60.75 $\mathrm{nGy}$ saat $^{-1}$ aralığında değiştiği ve ortalama değerin $34.31 \mathrm{nGy}$ saat $^{-1}$ olduğu belirlenmiştir. Bu ortalama değerin Dünya ortalamas1 değerinden (59 nGy saat $\left.^{-1}\right)$ (UNSCEAR, 2000) daha düşük olduğu gözlemlenmiştir. $\mathrm{H}_{\mathrm{ex}}$ değerlerinin $0.09-0.34$ aralığında değiştiği ve ortalama değerin 0.19 olduğu belirlenmiştir. 
Tablo 3. Karayemişin tüketimine bağlı olarak ${ }^{226} \mathrm{Ra},{ }^{232} \mathrm{Th},{ }^{137} \mathrm{Cs}$ ve ${ }^{40} \mathrm{~K}$ 'ın günlük alımı, y1llık alınan etkin doz ve kanserojen risk değerleri.

\begin{tabular}{|c|c|c|c|c|c|c|c|c|c|c|c|c|c|}
\hline \multirow{2}{*}{ İstasyon } & \multicolumn{4}{|c|}{$\begin{array}{l}\text { Radyonüklidlerin Günlük Alımı } \\
\left.\text { GA (Bq gün }{ }^{-1}\right)\end{array}$} & \multicolumn{4}{|c|}{$\begin{array}{l}\text { Yıllık Alınan Etkin Doz } \\
\text { YAED }\left(\mu \mathrm{Sv} \mathrm{yll}^{-1}\right)\end{array}$} & \multirow{2}{*}{$\underset{\left(\mu \mathrm{Sv} \mathrm{yll}^{-1}\right)}{\mathrm{YAED}_{\mathrm{T}}}$} & \multicolumn{4}{|c|}{ Kanserojen Risk (KR) } \\
\hline & ${ }^{226} \mathbf{R a}$ & ${ }^{232} \mathrm{Th}$ & ${ }^{137} \mathrm{Cs}$ & ${ }^{40} \mathbf{K}$ & ${ }^{226} \mathbf{R a}$ & ${ }^{232} \mathrm{Th}$ & ${ }^{137} \mathrm{Cs}$ & ${ }^{40} \mathbf{K}$ & & $\begin{array}{l}{ }^{226} \mathbf{R a} \\
\left(\times 10^{-6}\right)\end{array}$ & $\begin{array}{l}{ }^{232} \mathrm{Th} \\
\left(\times 10^{-7}\right)\end{array}$ & $\begin{array}{l}{ }^{137} \mathrm{Cs} \\
\left(\times 10^{-10}\right)\end{array}$ & $\begin{array}{l}{ }^{40} \mathrm{~K} \\
\left(\times 10^{-5}\right)\end{array}$ \\
\hline \multicolumn{14}{|l|}{ Trabzon } \\
\hline Yomra & - & 0.002 & 0.002 & 0.620 & - & 0.20 & 0.01 & 1.40 & 1.61 & - & 1.64 & 0.55 & 1.04 \\
\hline Araklı & 0.007 & 0.002 & - & 0.590 & 0.75 & 0.20 & - & 1.33 & 2.28 & 1.99 & 1.68 & - & 0.99 \\
\hline Çamburnu & 0.001 & 0.002 & - & 0.485 & 0.06 & 0.18 & - & 1.10 & 1.34 & 0.16 & 1.47 & - & 0.81 \\
\hline Sürmene & 0.001 & 0.004 & - & 0.740 & 0.11 & 0.33 & - & 1.67 & 2.12 & 0.29 & 2.75 & - & 1.24 \\
\hline Of & - & - & - & 0.486 & - & - & - & 1.10 & 1.10 & - & - & - & 0.81 \\
\hline \multicolumn{14}{|l|}{ Rize } \\
\hline İyidere & 0.001 & - & 0.003 & 0.720 & 0.12 & - & 0.02 & 1.63 & 1.77 & 0.32 & - & 0.89 & 1.21 \\
\hline Derepazarı & 0.001 & 0.003 & 0.001 & 0.500 & 0.12 & 0.22 & 0.01 & 1.13 & 1.47 & 0.32 & 1.82 & 0.27 & 0.84 \\
\hline Rize Merkez & - & - & 0.028 & 0.491 & - & - & 0.13 & 1.11 & 1.24 & - & - & 7.42 & 0.82 \\
\hline Çayeli & - & 0.003 & 0.010 & 0.713 & - & 0.22 & 0.05 & 1.61 & 1.88 & - & 1.80 & 2.50 & 1.20 \\
\hline Pazar & 0.014 & 0.002 & 0.001 & 0.643 & 1.45 & 0.20 & 0.00 & 1.46 & 3.11 & 3.86 & 1.66 & 0.19 & 1.08 \\
\hline Ardeşen & 0.005 & - & 0.002 & 0.668 & 0.55 & - & 0.01 & 1.51 & 2.07 & 1.48 & - & 0.49 & 1.12 \\
\hline Findıklı & 0.002 & 0.001 & 0.005 & 0.468 & 0.25 & 0.10 & 0.02 & 1.06 & 1.43 & 0.67 & 0.82 & 1.32 & 0.78 \\
\hline \multicolumn{14}{|l|}{ Artvin } \\
\hline Arhavi & 0.002 & - & 0.001 & 0.609 & 0.25 & - & 0.01 & 1.38 & 1.63 & 0.65 & - & 0.37 & 1.02 \\
\hline Нора & 0.012 & 0.006 & 0.003 & 0.530 & 1.25 & 0.50 & 0.02 & 1.20 & 2.96 & 3.32 & 4.17 & 0.90 & 0.89 \\
\hline $\begin{array}{l}\text { Dünya } \\
\text { Ortalamas1 }\end{array}$ & & & & & $120^{*}$ & $120^{*}$ & & $170^{*}$ & $290^{*}$ & & & & \\
\hline
\end{tabular}

*UNSCEAR, 2000; ** Khandaker vd., 2015.

Tablo 4. Toprak örneklerinde ${ }^{226} \mathrm{Ra},{ }^{232} \mathrm{Th},{ }^{137} \mathrm{Cs}$ ve ${ }^{40} \mathrm{~K}$ 'ın aktivite konsantrasyonları, radyum eşdeğerleri, soğurulan doz oranları, dış tehlike indeksleri ve yıllık etkin doz oranları.

\begin{tabular}{|c|c|c|c|c|c|c|c|c|}
\hline \multirow{2}{*}{ İstasyon } & \multicolumn{4}{|c|}{ Aktivite Konsantrasyonları (Bq kg $\left.{ }^{-1}\right)$} & \multirow{2}{*}{$\underset{\left(\mathbf{B q} \mathbf{k g}^{-1}\right)}{\mathbf{R a}_{\mathrm{eq}}}$} & \multirow{2}{*}{$\begin{array}{c}\text { D } \\
(\text { nGy saat } \\
\end{array}$} & \multirow{2}{*}{$\mathbf{H}_{\mathrm{ex}}$} & \multirow{2}{*}{$\begin{array}{c}\text { YED } \\
\left(\mu \mathrm{Sv} \mathrm{yll}^{-1}\right)\end{array}$} \\
\hline & ${ }^{226} \mathbf{R a}$ & ${ }^{232} \mathrm{Th}$ & ${ }^{137} \mathrm{Cs}$ & ${ }^{40} \mathbf{K}$ & & & & \\
\hline \multicolumn{9}{|l|}{ Trabzon } \\
\hline Yomra & $7.34 \pm 0.39$ & $9.04 \pm 0.58$ & $22.67 \pm 1.04$ & $506.28 \pm 21.52$ & 59.25 & 29.96 & 0.16 & 36.75 \\
\hline Araklı & $32.93 \pm 1.28$ & $30.45 \pm 1.69$ & $100.67 \pm 5.23$ & $650.94 \pm 38.52$ & 126.60 & 60.75 & 0.34 & 74.50 \\
\hline Çamburnu & $16.24 \pm 0.82$ & $18.21 \pm 1.12$ & $10.48 \pm 0.46$ & $280.14 \pm 10.78$ & 63.85 & 30.18 & 0.17 & 37.02 \\
\hline Sürmene & $10.56 \pm 0.42$ & $17.45 \pm 0.92$ & $2.80 \pm 0.15$ & $441.22 \pm 20.51$ & 69.49 & 33.82 & 0.19 & 41.47 \\
\hline Of & $12.15 \pm 0.67$ & $17.49 \pm 1.08$ & $27.31 \pm 1.32$ & $241.42 \pm 12.31$ & 55.75 & 26.24 & 0.15 & 32.19 \\
\hline \multicolumn{9}{|l|}{ Rize } \\
\hline İyidere & $29.73 \pm 1.41$ & $40.65 \pm 1.74$ & $4.53 \pm 0.21$ & $368.95 \pm 15.49$ & 116.27 & 53.67 & 0.31 & 65.82 \\
\hline Derepazarı & $21.04 \pm 0.86$ & $27.98 \pm 1.35$ & $51.70 \pm 2.84$ & $473.36 \pm 22.48$ & 97.50 & 46.36 & 0.26 & 56.86 \\
\hline Rize Merkez & $30.27 \pm 1.77$ & $42.62 \pm 1.84$ & $368.11 \pm 20.24$ & $205.67 \pm 11.32$ & 107.05 & 48.30 & 0.29 & 59.24 \\
\hline Çayeli & $5.40 \pm 0.33$ & $10.73 \pm 0.57$ & $100.61 \pm 4.72$ & $204.84 \pm 8.81$ & 36.52 & 17.52 & 0.10 & 21.48 \\
\hline Pazar & $19.28 \pm 0.88$ & $14.18 \pm 0.83$ & $15.97 \pm 0.82$ & $146.66 \pm 5.82$ & 50.85 & 23.59 & 0.14 & 28.93 \\
\hline Ardeşen & $6.89 \pm 0.37$ & $9.17 \pm 0.62$ & $203.91 \pm 9.03$ & $180.19 \pm 8.12$ & 33.88 & 16.24 & 0.09 & 19.91 \\
\hline Findiklı & $30.34 \pm 1.43$ & $37.89 \pm 1.87$ & $9.75 \pm 0.42$ & $290.59 \pm 11.94$ & 106.90 & 49.02 & 0.29 & 60.12 \\
\hline \multicolumn{9}{|l|}{ Artvin } \\
\hline Arhavi & $16.66 \pm 0.83$ & $18.63 \pm 1.25$ & $62.07 \pm 3.07$ & $148.34 \pm 7.84$ & 54.72 & 25.14 & 0.15 & 30.83 \\
\hline Hopa & $5.21 \pm 0.25$ & $6.18 \pm 0.32$ & $188.57 \pm 7.46$ & $321.51 \pm 15.69$ & 38.80 & 19.55 & 0.10 & 23.97 \\
\hline Ortalama & 17.43 & 21.48 & 83.51 & 318.58 & 72.67 & 34.31 & 0.19 & 42.08 \\
\hline Minimum & 5.21 & 6.18 & 2.80 & 146.66 & 33.88 & 16.24 & 0.09 & 19.91 \\
\hline Maksimum & 32.93 & 42.62 & 368.11 & 650.94 & 126.60 & 60.75 & 0.34 & 74.50 \\
\hline Dünya Ortalaması* & 35 & 30 & & 400 & $370^{\mathrm{a}}$ & 59 & & 70 \\
\hline
\end{tabular}

*UNSCEAR, 2000; ${ }^{a}$ Sinırdeğer 
Tahmin edilen $\mathrm{H}_{\mathrm{ex}}$ değerlerinin 1 den küçük olduğu tespit edildi. Yıllık etkin doz değerlerinin ise $19.91-74.50 \mu \mathrm{Sv}_{1} 1^{-1}$ aralığında değiştiği ve ortalama değerin $42.08 \mu \mathrm{Sv} \quad \mathrm{y}_{1} 1^{-1}$ olduğu belirlenmiştir. $\mathrm{Bu}$ ortalama değerin Dünya ortalaması değerinden $\left(70 \mu \mathrm{Sv} \mathrm{y}_{1} 1^{-1}\right)$ (UNSCEAR, 2000) daha düşük olduğu tespit edilmiştir.

Topraktan bitki kökleri boyunca karayemişin meyve kısmına geçen ${ }^{226} \mathrm{Ra},{ }^{232} \mathrm{Th},{ }^{137} \mathrm{Cs}$ ve ${ }^{40} \mathrm{~K}$ radyonüklidleri için hesaplanan transfer faktörleri Tablo 5'de verilmiştir. Transfer faktörü, radyoaktif kirlilik gösteren topraklardaki karasal bitkilerin bitkisel arıtma potansiyelinin önemli bir göstergesidir (Kaewtubtim vd., 2017). Transfer faktörü ayrıca ürün bitkilerine radyonüklidlerin geçişini belirlemek içinde kullanılır (Aung vd., 2015). TF değerleri 1'den büyükse $(\mathrm{TF}>1)$ topraktan bitkiye radyonüklidlerin geçişinin yüksek olduğunu gösterir (Baker ve Brooks, 1989; $\mathrm{Hu}$ vd., 2014). TF değerleri ${ }^{226} \mathrm{Ra}$ için $0.013-$ $0.854,{ }^{232} \mathrm{Th}$ için $0.011-0.353,{ }^{137} \mathrm{Cs}$ için $0.003-$ 0.274 ve ${ }^{40} \mathrm{~K}$ için $0.33-1.60$ aralığında değiştiği gözlemlenmiş ve ortalama değerlerinin sırasıyla $0.166,0.088,0.060$ ve 0.83 olarak hesaplanmıştır.

Tablo 5. Topraktan karayemişin meyve kısmına ${ }^{226} \mathrm{Ra},{ }^{232} \mathrm{Th},{ }^{137} \mathrm{Cs}$ ve ${ }^{40} \mathrm{~K}$ 'ın transfer faktörleri.

\begin{tabular}{|c|c|c|c|c|}
\hline \multirow{2}{*}{ İstasyon } & \multicolumn{4}{|c|}{ Transfer faktörü (TF) } \\
\hline & ${ }^{226} \mathrm{Ra}$ & ${ }^{232} \mathrm{Th}$ & ${ }^{137} \mathrm{Cs}$ & ${ }^{40} \mathrm{~K}$ \\
\hline \multicolumn{5}{|l|}{ Trabzon } \\
\hline Yomra & - & 0.095 & 0.033 & 0.45 \\
\hline Arakli & 0.081 & 0.029 & - & 0.33 \\
\hline Çamburnu & 0.013 & 0.042 & - & 0.63 \\
\hline Sürmene & 0.037 & 0.082 & - & 0.61 \\
\hline Of & - & - & - & 0.73 \\
\hline \multicolumn{5}{|l|}{ Rize } \\
\hline İyidere & 0.014 & - & 0.274 & 0.71 \\
\hline Derepazarı & 0.020 & 0.034 & 0.007 & 0.38 \\
\hline Rize Merkez & - & - & 0.028 & 0.87 \\
\hline Çayeli & - & 0.088 & 0.034 & 1.27 \\
\hline Pazar & 0.268 & 0.061 & 0.017 & 1.60 \\
\hline Ardeşen & 0.287 & - & 0.003 & 1.35 \\
\hline Findiklı & 0.030 & 0.011 & 0.188 & 0.59 \\
\hline \multicolumn{5}{|l|}{ Artvin } \\
\hline Arhavi & 0.053 & - & 0.008 & 1.50 \\
\hline Hopa & 0.854 & 0.353 & 0.007 & 0.60 \\
\hline Ortalama & 0.166 & 0.088 & 0.060 & 0.83 \\
\hline Min.-Mak. & $0.013-0.854$ & $0.011-0.353$ & $0.003-0.274$ & $0.33-1.60$ \\
\hline
\end{tabular}

\section{Sonuçlar}

Doğu Karadeniz Bölgesi'nde on dört farklı istasyondan toplanan karayemiş örneklerinin meyve kısmında ve bu türün köklerindeki toprak örneklerinde doğal $\left({ }^{226} \mathrm{Ra},{ }^{232} \mathrm{Th}\right.$ ve $\left.{ }^{40} \mathrm{~K}\right)$ ve yapay $\left({ }^{137} \mathrm{Cs}\right)$ radyonüklidlerin aktivite konsantrasyonları gama 1şını spektroskopisi kullanılarak belirlenmiştir. Karayemişin meyve kısmında ve toprak örneklerinde ortalama aktivite konsantrasyonları sırasıyla ${ }^{226} \mathrm{Ra}$ için 1.75 ve $17.43 \mathrm{~Bq} \mathrm{~kg}^{-1},{ }^{232} \mathrm{Th}$ için 1.03 ve $21.48 \mathrm{~Bq} \mathrm{~kg}^{-1}$, ${ }^{137} \mathrm{Cs}$ için 2.07 ve $83.51 \mathrm{~Bq} \mathrm{~kg}^{-1}$ ve ${ }^{40} \mathrm{~K}$ için 215.38 ve $318.58 \mathrm{~Bq} \mathrm{~kg}^{-}$ ${ }^{1}$ olarak belirlenmiştir. Toprak örnekleri için elde edilen ${ }^{226} \mathrm{Ra},{ }^{232} \mathrm{Th}$ ve ${ }^{40} \mathrm{~K}$ ' $1 \mathrm{n}$ ortalama aktivite konsantrasyonları Dünya ortalaması değerlerinden daha düşük olduğu tespit edilmiştir. Karayemişin tüketilmesiyle radyonüklidlerin vücuda alınmasından dolay1 insana geçen ${ }^{226} \mathrm{Ra},{ }^{232} \mathrm{Th},{ }^{137} \mathrm{Cs}$ ve ${ }^{40} \mathrm{~K}$ 'ın günlük alımı, yıllık alınan etkin doz ve 
kanserojen risk değerleri uluslararası değerlerden daha düşük olarak hesaplanmıştır. Ayrıca, toprak örnekleri için radyum eşdeğer-aktivitesi, soğurulan doz oranı, dış tehlike indeksi ve yıllık etkin doz eşdeğerinin ortalama değerleri sırasıyla $72.67 \mathrm{~Bq} \mathrm{~kg}^{-1}, 34.31 \mathrm{nGy}$ saat $^{-1}, 0.19$ ve 42.08 $\mu \mathrm{Sv} \mathrm{y}^{-1}{ }^{-1}$ olarak hesaplanmış ve tavsiye edilen uluslararası değerler ile karşılaştırılmıştır. Bunlara ilaveten topraktan karayemişin meyve kısmına geçen ${ }^{226} \mathrm{Ra},{ }^{232} \mathrm{Th},{ }^{137} \mathrm{Cs}$ ve ${ }^{40} \mathrm{~K}$ radyonüklidleri için transfer faktörlerinin ortalama değerleri sirasiyla $0.166,0.088,0.060$ ve 0.83 olarak hesaplanmıştır. $\mathrm{Bu}$ çalışmadan elde edilen sonuçlar, ortalama doz oranlarının tavsiye edilen Dünya ortalaması değerlerini aşmadığını ve halk sağlığı için herhangi bir risk oluşturmadığını ortaya koymaktadır.

\section{Kaynaklar}

Alasalvar, C., Al-Farsi, M. ve Shahidi, F., 2005. Compositional characteristics and antioxidant components of cherry laurel varieties and pekmez, Journal of Food Science, 70, 1, 47-52.

Amanjeet, Kumar, A., Kumar, S., Singh, J., Singh, P. ve Bajwa, B.S., 2017. Assessment of natural radioactivity levels and associated dose rates in soil samples from historical city Panipat, India, Journal of Radiation Research and Applied Sciences, 10, 283-288.

Amrani, D. ve Tahtat, M., 2001. Natural radioactivity in Algerian building materials, Applied Radiation and Isotopes, 54, 4, 687-689.

Aung, H.P., Aye, Y.S., Mensah, A.D., Omari, R.A., Djedidi, S., Oikawa, Y., Ohkama-Ohtsu, N., Yokoyama, T. ve Bellingrath-Kimura, S.D., 2015. Relations of fine-root morphology on ${ }^{137} \mathrm{Cs}$ uptake by fourteen Brassica species, Journal of Environmental Radioactivity, 150, 203-212.

Badran, H.M., Sharshar, T. ve Elnimer, T., 2003. Levels of ${ }^{137} \mathrm{Cs}$ and ${ }^{40} \mathrm{~K}$ in edible parts of some vegetables consumed in Egypt, Journal of Environmental Radioactivity, 67, 181-190.

Baker, A.J.M. ve Brooks, R.R., 1989. Terrestrial higher plants which hyperaccumulate metallic elements - a review of their distribution, ecology and phytochemistry, Biorecovery, 1, 81-126.

Baltas, H., Kiris, E. ve Sirin, M., 2017. Determination of radioactivity levels and heavy metal concentrations in seawater, sediment and anchovy (Engraulis encrasicolus) from the Black Sea in Rize, Turkey, Marine Pollution Bulletin, 116, 528-533.
Baltas, H., Kiris, E., Ustabas, I., Yilmaz, E., Sirin, M., Kuloglu, E. ve Erdogan Gunes, B., 2014. Determination of natural radioactivity levels of some concretes and mineral admixtures in Turkey, Asian Journal of Chemistry, 26, 13, 3946-3952.

Beretka, J. ve Mathew, P.J., 1985. Natural radioactivity of Australian building materials, waste and byproducts, Health Physics, 48, 87-95.

Celik, N., Cevik, U., Celik, A. ve Koz, B., 2009. Natural and artificial radioactivity measurements in Eastern Black Sea region of Turkey, Journal of Hazardous Materials, 162, 146-153.

Celik, N., Cevik, U., Celik, A. ve Kucukomeroglu, B., 2008. Determination of indoor radon and soil radioactivity levels in Giresun, Turkey, Journal of Environmental Radioactivity, 99, 1349-1354.

Chen, S.B., Zhu, Y.G. ve Hu, Q.H., 2005. Soil to plant transfer of ${ }^{238} \mathrm{U},{ }^{226} \mathrm{Ra}$ and ${ }^{232} \mathrm{Th}$ on a uranium mining-impacted soil from southeastern China, Journal of Environmental Radioactivity, 82,223-236.

Currie, L.A., 1968. Limits for qualitative detection and quantitative determination. Application to radiochemistry, Analytical Chemistry, 40, 586593.

Hu, N., Ding, D., Li, G., Zheng, J., Li, L., Zhao, W. ve Wang, Y., 2014. Vegetation composition and ${ }^{226} \mathrm{Ra}$ uptake by native plant species at a uranium mill tailings impoundment in South China, Journal of Environmental Radioactivity, 129, 100-106.

IAEA (International Atomic Energy Agency), 2011. Radiation Protection and Safety of Radiation Sources: International Basic Safety Standards. IAEA Safety standards series no. GSR Part 3 (Interim), STI/PUB/1531, pp. 190-219.

IUR (International Union of Radio ecologists), 1994. Handbook of parameter values for the prediction of radionuclide transfer in temperate environments, Technical reports series no. 364, Vienna: International Atomic Energy Agency (IAEA).

Kaewtubtim, P., Meeinkuirt, W., Seepom, S. ve Pichtel, J., 2017. Radionuclide $\left({ }^{226} \mathrm{Ra},{ }^{232} \mathrm{Th}\right.$, ${ }^{40} \mathrm{~K}$ ) accumulation among plant species in mangrove ecosystems of Pattani Bay, Thailand, Marine Pollution Bulletin, 115, 391-400.

Keser, R., Korkmaz Görür, F., Akçay, N. ve Okumuşoğlu, N.T., 2011. Radionuclide concentration in tea, cabbage, orange, kiwi and soil and lifetime cancer risk due to gamma 
radioactivity in Rize, Turkey, Journal of the Science of Food and Agriculture, 91, 987-991.

Khandaker, M.U., Asaduzzaman, Kh., Nawi, S.M., Usman, A.R., Amin, Y.M., Daar, E., Bradley, D.A., Ahmed, H. ve Okhunov, A.A., 2015. Assessment of radiation and heavy metals risk due to the dietary intake of marine fishes (Rastrelliger kanagurta) from the Straits of Malacca. PLOS ONE 10,6, 1-16.

Khandaker, M.U., Norfadira, B.W., Amin, Y.M. ve Bradley, D.A., 2013. Committed effective dose from naturally occurring radionuclides in shellfish, Radiation Physics and Chemistry, 88, $1-6$.

Kolayli, S., Küçük, M., Duran, C., Candan, F. ve Dinçer, B., 2003. Chemical and antioxidant properties of Laurocerasus officinalis Roem. (cherry laurel) fruit grown in the Black Sea region, Journal of Agricultural and Food Chemistry, 51, 25, 7489-7494.

Korkmaz Görür, F., Keser, R., Akçay, N., Dizman, S., As, N. ve Okumuşoğlu, N.T., 2012. Radioactivity and heavy metal concentrations in food samples from Rize, Turkey, Journal of the Science of Food and Agriculture, 92, 307-312.

Krieger, R., 1981. Radioactivity of construction materials, Betonwerk Fertigteil Technology, 47, 8, 468-473.

Kurnaz, A., Kucukomeroglu, B., Damla, N. ve Cevik, U., 2011. Radiological maps for Trabzon, Turkey, Journal of Environmental Radioactivity, 102, 393-399.

Kurnaz, A., Küçükömeroğlu, B., Keser, R., Okumusoglu, N.T., Korkmaz, F., Karahan, G. ve Çevik, U., 2007. Determination of radioactivity levels and hazards of soil and sediment samples in Firtına Valley (Rize, Turkey), Applied Radiation and Isotopes, 65, 1281-1289.

Lauria, D.C., Ribeiro, F.C.A., Conti, C.C. ve Loureiro, F.A., 2009. Radium and uranium levels in vegetables grown using different farming management systems, Journal of Environmental Radioactivity, 100, 176-183.

Liyana-Pathirana, C.M., Shahidi, F. ve Alasalvar, C., 2006. Antioxidant activity of cherry laurel fruit (Laurocerasus officinalis Roem.) and its concentrated juice, Food Chemistry, 99, 1, $121-128$.
Lu, J., Huang, Y., Li, F., Wang, L., Li, S. ve Hsia, Y., 2006. The investigation of ${ }^{137} \mathrm{Cs}$ and ${ }^{90} \mathrm{Sr}$ background radiation levels in soil and plant around Tianwan NPP, China, Journal of Environmental Radioactivity, 90, 89-99.

Persson, B.R.R. ve Holm, E., 2011. Polonium-210 and lead-210 in the terrestrial environment: a historical review, Journal of Environmental Radioactivity, 102, 420-429.

Pulhani, V.A., Dafauti, S., Hegde, A.G., Sharma, R.M. ve Mishra, U.C., 2005. Uptake and distribution of natural radioactivity in wheat plants from soil, Journal of Environmental Radioactivity, 79, 331-346.

Shanthi, G., Kumaran, J.T.T., Raj, G.A.G. ve Maniyan, C.G., 2012. Transfer factor of the radionuclides in food crops from high-background radiation area of south west India, Radiation Protection Dosimetry, 149, 3, 327-332.

Strode, S.A., Ott, L.E., Pawson, S. ve Bowyer, T.W., 2012. Emission and transport of cesium-137 from boreal biomass burning in the summer of 2010, Journal of Geophysical Research, 117, 18.

Tzortzis, M., Svoukis, E. ve Tsetos, H., 2004. A comprehensive study of natural gamma radioactivity levels and associated dose rates from surface soils in Cyprus, Radiation Protection Dosimetry, 109, 217-224.

UNSCEAR (United Nations Scientific Committee on the Effect of Atomic Radiation), 2000. Radiation sources and Effects of ionizing radiation. Report to General Assembly, with Scientific Annexes. United Nations, New York.

UNSCEAR (United Nations Scientific Committee on the Effects of Atomic Radiation), 1982. Report of the United Nations Scientific Committee on the Effects of Atomic Radiation to the General Assembly. United Nations, New York.

USEPA (US Energy Protection Agency), 1999. Cancer risk coefficients for environmental exposureto radionuclides. Federal Guidance Report No.13;EPA 402-R-99-001.

Yazici, K., Ertuğral, B., Damla, N. ve Apaydın, G., 2008. Radioactive Contamination in Lichens Collected from Trabzon and Rize in the Eastern Black Sea Region, Turkey, and a Comparison with that of 1995, Bulletin of Environmental Contamination and Toxicology, 80, 475-479. 